\title{
ON THE SuITABILITY OF USING ANT COLONY OPTIMIZATION FOR ROUTING MULTIMEDIA CONTENT OVER WIRELESS SENSOR NETWORKS
}

\author{
Hiba Al-Zurba ${ }^{1}$, Taha Landolsi ${ }^{1}$, Mohamed Hassan $^{2}$, and Fouad Abdelaziz ${ }^{3}$ \\ ${ }^{1}$ Department of Computer Science and Engineering, American University of Sharjah, \\ United Arab Emirates \\ tlandolsieaus.edu \\ ${ }^{2}$ Department of Electrical Engineering, American University of Sharjah, United Arab \\ Emirates \\ mshassaneaus. edu \\ ${ }^{3}$ Engineering Systems Management Graduate Program, American University of Sharjah, \\ United Arab Emirates \\ fabdelaziz@aus.edu
}

\begin{abstract}
This paper studies the suitability of using a meta-heuristic ant colony technique in routing multimedia content over wireless sensor networks. The presented technique is both energy and QoS-aware. Ant colony algorithm is used to find the optimal routing path. Optimality is in the sense of minimizing energy consumption and increasing link quality and reliability. The proposed approach results in minimizing energy consumption and prolonging the lifetime of the network. Moreover, the optimal path has a high link quality and reliability which enhances video frame quality and ensures high probability of successful delivery of video frames. The importance given to energy consumption, link quality, and link reliability metrics can be varied depending on the multimedia application requirements.
\end{abstract}

\section{KEYWORDS}

Wireless Sensor Networks, Multimedia communications, Ant colony optimization.

\section{INTRODUCTION}

Wireless Sensor Networks (WSNs) have captured the attention of researchers over the past decade because of the diverse applications they support and the flexibility of network deployment options they provide. These advantages, along with the remarkable advances in sensor technology, made WSNs a viable option for many tracking and monitoring applications [1-5] even in mission-critical situations such as battlefield troops' deployment, and search and rescue operations. In such applications, sensors nodes can collaboratively monitor the network environment and report real-time information about the monitored phenomenon. Using sensor nodes with multimedia capabilities such as video cameras enhances the interpretation of the coverage area.

The deployment of WSN, however, does generally pose several challenges such as the limited energy sources for the sensing nodes which shortens the network lifetime, the relatively short communication range which reduces the network coverage area, the limited processing and

DOI : 10.5121/jgraphoc.2011.3202 
International journal on applications of graph theory in wireless ad hoc networks and sensor networks (GRAPH-HOC) Vol.3, No.2, June 2011

storage capability of sensor nodes which imposes frequent backhauling of acquired data, and the quality of service (QoS) provisioning under harsh network conditions [2]. These challenges and constraints are much more stringent in multimedia WSNs (MWSNs) [3-5]. Two main challenges faced in MWSNs are quality of service (QoS) requirements and energy availability [10-13].

Many routing protocols with various metrics have been developed for WSNs but only few considered the particularities of MWSN [30-35]. In general, routing protocols in WSN assume structured network architecture where deployment of nodes is pre-planned. Limited research has been done on routing protocol for MWSN deployed in ad-hoc manner that meets QoS requirements and at the same time considers energy efficiency for the purpose of prolonging the lifetime of the network. Common routing protocols classification is based on the network structure such as flat, hierarchical, location-based routing or classified based on the protocol operations such as multipath-based, query-based, negotiation-based, QoS-based, and coherentbased [36-40].

In the following discussion, we give a brief summary of the various routing protocols and techniques proposed for WSN.

The authors in [6] explore a group of routing protocols that are based on one or both of the following approaches: data centric and energy efficient approaches. In [7], the authors discussed various WSN routing protocols that are classified into data-centric, hierarchical, and locationbased routing.

In [8], many WSN routing techniques are discussed that are classified into categories based on the network structure and based on protocol operation. Protocols classified based on the network structure are divided into three groups: flat, hierarchical, and location-based routing. In Flat architecture, all nodes have the same responsibilities and tasks to perform. While in hierarchical architecture, nodes are clustered and clustered nodes do some processing, aggregation, and reduction of data in order to reduce power consumption. Location-based protocol depends on the position of nodes when relaying data to specific nodes rather than all network nodes. Protocols classified based on the network operation are divided into the following groups: multipath-based, query-based, negotiation-based, QoS-based, and coherent-based. It is evident in [7-8] that QoSbased routing protocols do not take into consideration power usage and energy aware routing protocols do not take into consideration QoS parameters.

A brief list of QoS-based routing protocols is given below:

- Sequential Assignment Routing (SAR) considers QoS in addition to energy efficiency, and packet priority when making a routing decision [14]. The advantages of this protocol are power efficiency, fault-tolerance, and easy recovery. However, the main disadvantage, especially when there are a large number of nodes, is the relatively high overhead caused by maintaining routing tables at each sensor node.

- SPEED provides soft real-time end-to-end guarantees [15]. It strives to guarantee a certain delivery speed for each packet in the network. Admission of packet at the network layer is based on the required speed. However, it does not consider energy consumption in its operation. 
International journal on applications of graph theory in wireless ad hoc networks and sensor networks (GRAPH-HOC) Vol.3, No.2, June 2011

- MMSPEED (multi-path and multi-speed) is an extension to SPEED protocol [16]. It is a multipath priority-based routing protocol. Based on the packet's reliability and timeliness requirement, a packet will be serviced with a certain QoS level and certain delivery speed. MMSPEED provides QoS differentiation in both reliability and timeliness domains. However, it does not handle the trade-off between energy and delay and it does not handle network layer aggregation.

- Energy-aware and QoS-based protocol finds a least cost energy efficient path and guarantees certain end-to-end delay [17]. While this protocol considers QoS and energy metrics, it only considers one real-time priority class which is only appropriate for a single real-time application and it is not suitable for multiple applications because it requires several priority classes for different real-time traffic

- Energy balanced routing metric (EBRM) is presented in [18]. It ensures reliable communication while balancing the network load and thus prolongs the network lifetime.

Here, we present a brief discussion of routing protocols that use ant colony optimization. Ant colony optimization is a meta-heuristic method used to solve difficult combinatorial optimization problems such as the travelling salesman problem (TSP), quadratic assignment problem (QAP), car sequencing problem, and network routing problem. Ant colony optimization is inspired by the behaviour of real ants seeking the path from their colony to the food source. Real ant colonies explore the possible paths between food source and their colony while depositing pheromones and afterwards find the shortest path by following the one with the highest pheromone trails.

There are several variations and extensions to the original Ant System (AS) algorithm used by Marco Dorigo that solve various computational problems based on a probabilistic approach. Some of these variants are Elitist Ant System, Rank-Based Ant System, Max-Min Ant System, and Ant Colony System (ACS) [19].

Some of ant colony based routing protocols for WSNs are discussed in [20-26]. In [20], the authors propose an ant colony based routing protocol called energy-efficient ant-based routing (EEABR) that finds optimal routing paths in terms of distance and energy between source nodes and sink nodes. This algorithm reduces the communication loads and saves energy which leads to extending the network lifetime. In [21-22], the authors propose a dynamic and reliable routing protocol using ant colony optimization algorithm. This protocol uses multipath data transmission to increase reliability while maximizing network life time. This protocol has a higher energy saving than EEABR. In [23], the authors present a self-optimized multipath routing protocol that considers energy level, delay, and velocity constraints. It also uses multipath approach to avoid congestions and in turns maximizes throughput and minimizes data loss. In [24], the authors propose an improved adaptive routing (IAR) algorithm that optimizes energy consumption, latency, throughput, and packet survival rate. However, these ant colony based routing protocols mentioned above do not consider multimedia QoS requirements such as stringent delay, delay jitter, loss rate, link quality, and link reliability in addition to minimizing energy consumption.

The authors in [25] proposes an extension to IAR called multimedia-enabled improved adaptive routing (M-IAR) that targets multimedia applications in WSN. M-IAR optimizes end-to-end delay and jitter in addition to minimizing energy consumption. However, this protocol minimizes energy consumption by choosing the path that includes the least number of hops and has the 
International journal on applications of graph theory in wireless ad hoc networks and sensor networks (GRAPH-HOC) Vol.3, No.2, June 2011

shortest distance. It does not take into consideration the remaining energy levels of the sensor nodes and therefore the lifetime of the network.

In this paper, an ant colony based routing protocol is developed for MWSN that is energy-aware and QoS-aware. This routing protocol uses ant colony optimization to find the routing path that maximizes the end-to-end path quality and reliability as well as the network lifetime. End-to-end delay is a constraint set depending on the application requirement. Each metric used in the path cost can be attributed an importance that varies depending on the application requirements.

The rest of the paper is organized as follows. Section 2 describes the proposed routing algorithm. Section 3 describes the simulation environment. Section 4 analyzes the proposed algorithm and discusses the results and the effect of changing various parameters. Section 4 analyzes the results of MWSN simulation. In the last section, the conclusion is drawn.

\section{ANT COLONY-BASEd Routing FOR MUltimedia WSN}

The proposed algorithm uses ant colony optimization technique to find the optimal path in WSNs. The optimal path is the one with the minimum cost in terms of energy consumption, link quality, and reliability. The end-to-end delay is set as a constraint when choosing the optimal path depending on the application requirements and the type of traffic transmitted.

\subsection{Cost Function in Ant Colony Algorithm for Multimedia WSN}

In this section, we derive a cost function that is suitable for routing multimedia content over WSN. We recognize that the cost $C_{i j}$ for any given $\operatorname{link}(i, j)$ should consider such elementary costs as link energy consumption $E_{i j}$, link quality $Q_{i j}$, and link reliability $R_{i j}$.

The energy consumption cost $E_{i j}$ is the summation of the communication energy $E_{c o m}$, the transmission energy $E_{t r}$, and the receiver node energy level $E_{l v l}$. The communication energy is usually written in the form $E_{c o m}=\kappa L_{i j}^{n}$, where $\kappa$ is a constant, to indicate that it is proportional to the link distance $L_{i j}$ raised to the path loss exponent, $n$. The path loss exponent is typically an empirical value that varies with the type of clutter and obstacles faced by the communication signal during propagation, ranging from $n=2$ in free-space line of sight links, to $4 \leq n \leq 6$ in cluttered urban environments [27][28]. The energy transmission cost $E_{t r}=E_{b} N_{b}$ is the packet size in bits $N_{b}$ multiplied by the energy consumed per bit transmission, $E_{b}$. The energy level cost $E_{l v l}=I-E_{j}$, is defined as the difference between the initial energy $I$ and the current energy $E_{j}$ of the receiving node $j$. Therefore, the receiving node $j$ with lower energy level has a higher cost and thus a lower probability to be forwarded any traffic from node $i$.

The energy consumption component of the cost function contains different energy consumption metrics. Based on the above definitions, it is clear that the path with the minimum cost is the one with the shortest distance which reduces communication energy cost. Moreover, taking into account the receiver node's energy level would result in prolonging the life time of the network. Each component of the energy consumption metric can be given different degrees of importance 
International journal on applications of graph theory in wireless ad hoc networks and sensor networks

(GRAPH-HOC) Vol.3, No.2, June 2011

to achieve a finer degree of control over the link energy consumption and, therefore, the overall network lifetime.

To take into consideration the link reliability, we let $r_{i j}$ be the percentage of time the link $(i, j)$ is up and functioning properly. The link reliability cost can, therefore, be defined as $R_{i j}=1-r_{i j}$, thereby giving the link with low reliability a higher cost and less likelihood to be chosen in any routing decision.

Finally, the link quality cost $Q_{i j}$ can be defined as the bit error rate on link $(i, j)$. Noting that for a given path composed of several links, the links quality and reliability are multiplicative and the links energy consumption is additive. Hence, a linearization transform can be used e.g. applying the logarithmic function to the links quality and reliability to make the overall link cost additive [29]. Under these assumptions, the overall cost for the link $(i, j)$ can be written as:

$$
C_{i j}=w_{1} \bar{E}_{i j}+w_{2} \bar{Q}_{i j}+w_{3} \bar{R}_{i j}
$$

where $\bar{E}_{i j}, \bar{Q}_{i j}, \bar{R}_{i j}$, are the normalized costs for the energy, quality, and reliability, respectively and $w_{1}, w_{2}, w_{3}$, are weights that accord more or less importance to a given component of the cost function, which is typically application-dependent.

\subsection{Ant Colony Optimization}

In ant colony optimization, each ant attempts to find the optimal path. In their journey from source node to destination node, ants move from node $i$ to a neighbouring node $j$ with a transition probability $P_{i j}$, defined as:

$$
P_{i j}[k]=\frac{\tau_{i j}{ }^{\alpha} \eta_{i j}{ }^{\beta}}{\sum_{l \in N_{i}[k]} \tau_{i l}{ }^{\alpha} \eta_{i l}{ }^{\beta}}, j \in N_{i}[k],
$$

where $P_{i j}[k]$ is the probability of ant $k$ to move from node $i$ to node $j, \tau_{i j}$ is the pheromone value deposited on the link $(i, j), \eta_{i j}$ is a heuristic value assigned to the link, and $(\alpha, \beta)$ are weights used to control the importance given to the pheromone and the heuristic values, respectively. Here, $N_{i j}[k]$ is the list of neighbours of node $i$ visited by ant $k$.

The pheromone value $\tau_{i j}$ on a given link depends on the likelihood that the ants pass by the link while constructing the solutions. The heuristic value $\eta_{i j}$, on the other hand, depends on the calculated cost of the link. The initial pheromone value is usually set to be equal for all links. The heuristic value of the link $(i, j)$ is the inverse of the link cost, $C_{i j}$. 
International journal on applications of graph theory in wireless ad hoc networks and sensor networks

(GRAPH-HOC) Vol.3, No.2, June 2011

\subsection{Ant Colony Algorithm Updates}

After all ants construct their solutions, the amount of pheromone value is updated only on the links of the paths found by all ants as follows:

$$
\tau_{i j}=\tau_{i j}+\sum_{k=1}^{m} \Delta \tau_{i j}[k], \forall(i, j) \in S[k]
$$

where $S[k]$ is the path found by ant $k$, and $\Delta \tau_{i j}[k]$ is the amount of pheromone update on link $(i, j)$ deposited by ant $k$ and can be computed as:

$$
\Delta \tau_{i j}[k]=\frac{1}{C_{s d}[k]}, \forall(i, j) \in S[k] .
$$

The total cost of a path is the summation of the cost of the intermediate links between a source $s$ and a destination $d: C_{s d}[k]=\sum_{s}^{d} C_{i j}$. The optimal path is the one with the minimum cost found by all ants. It is important to note that the pheromone value evaporates at a rate $\rho$ on all links in the paths constructed by all ants as follows:

$$
\tau_{i j}=\tau_{i j}(1-\rho), \forall(i, j) \in S[k]
$$

Every sensor node has its initial energy set to the same value $I$. For every packet transmitted, the energy of the transmitting node is reduced by [17][18]:

$$
\Delta E_{i j}=N_{b}\left(E_{b}+\varepsilon \cdot L_{i j}^{n}\right)
$$

where the transmission energy per bit is assumed to be $E_{b}=50 \mathrm{~nJ} / \mathrm{bit}$, and the transmitter amplifier parameter is $\varepsilon=10 \mathrm{pJ} / \mathrm{bit} / \mathrm{m}^{2}$.

\subsection{Ant Colony Algorithm}

In ant colony optimization algorithm, there are many parameters such as the number of ants $m$, the weights of $\alpha$ and $\beta$, and evaporation rate $\rho$. These parameters are set at the beginning of the algorithm. The cost of each link in the network is calculated based on the cost function we discussed earlier and fed back to the ant colony system. The algorithm can run for iterations. Every iteration, solutions are constructed by artificial ants, pheromones are updated, and the best path is chosen.

The following is a summary of ant colony optimization algorithm: 
International journal on applications of graph theory in wireless ad hoc networks and sensor networks

(GRAPH-HOC) Vol.3, No.2, June 2011

\author{
BEGIN \\ Input $m, \alpha, \beta, \rho$ \\ Set ant colony configuration \\ Set initial pheromone value and heuristic value \\ Get ant colony optimization system based on the calculated cost matrix \\ FOR $i=1$ to $n$th path $\mathbf{D O}$ \\ FOR $r=1$ to iterations DO \\ Reset ants \\ Construct ants' solutions \\ Apply local search \\ Update best path for $i$ \\ Update pheromones \\ ENDFOR \\ ENDFOR \\ Choose best path for $i$ \\ END
}

\title{
3. SimUlation
}

In this paper we simulated a multimedia WSN with $N_{S}$ sensor nodes and $S$ sink nodes. The sensor nodes are scattered in an ad-hoc manner to cover an area of interest e.g. a military battlefield that is $L \mathrm{~km}$ long by $W \mathrm{~km}$ wide. The aim is to monitor the area where sporadic events might happen, casualties might fall, and help and rescue operations might be needed. The sensors nodes with multimedia capabilities e.g. the body-area network carried over troops uniforms are assumed to know their physical location (e.g. GPS-enabled), broadcasting it periodically to other sensor nodes using small control packets. When an event takes place in the battlefield the sensor nodes in the vicinity will monitor the situation and transmit live video sequences of the area to the sink nodes. Sink nodes such as temporary field headquarters are assumed to have higher processing capabilities, larger transmission range, higher transmission bit rate, and ampler energy levels than sensor nodes.

In our simulation, events are generated randomly from random sensor nodes at a generation rate $\lambda$ event/s. For every event, video frames are generated from the source node at an encoding rate $F_{p}$ and are then packetized. Deadlines are set for every video frame and optimal paths are found using the proposed ant colony optimization algorithm. If a video frame arrives after the deadline, it would miss its scheduled play back time at the receiver node. In such a case the delayed frame will be of no use or even harmful and thus should be discarded by the sink node. If such scenario occurred, it would result in an unnecessary usage of network resources and nodes energy. To avoid transmitting packets which would be discarded later on, a calculation of the probability for this packet to meet the deadline is performed by the transmitting sensor node before transmitting any packets. Every node keeps track of its average queuing delay and the packet remaining delay is calculated based on the remaining propagation, remaining transmission, and total average queuing delay in the path. If the packet will not meet the deadline based on calculating the remaining delay, the packet is dropped. Due to the importance of meeting deadlines and to reduce packet loss, transmission of packets at each sensor node's queue is set to be based on earliest deadline first as shown in Figure 1. 


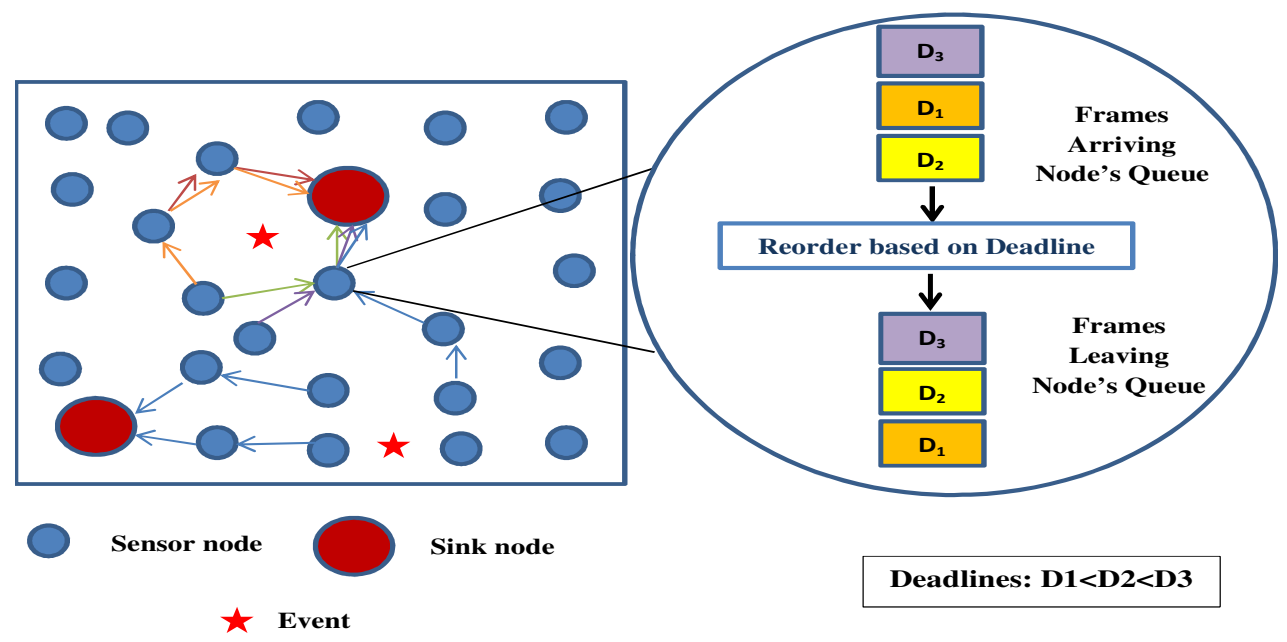

Figure 1. Simulation environment and assumptions for the multimedia WSN.

\section{Tuning of the Ant Colony Algorithm Parameters}

There are many parameters involved in any ant colony optimization problem such as the number of ants $m$, the weights of $\alpha$ and $\beta$, and evaporation rate $\rho$. In what follows, a study of the impact of these parameters on the time it takes to find the optimal path is explored. To do so, our simulation is run with different numbers of nodes. In each study, three iterations are executed for each run and the simulation is run 100 times to satisfy a $95 \%$ confidence interval.

\subsection{Choice of the Number of Ants}

The number of ants is usually set depending on the number of nodes in the WSN. Based on the results of the program runs, it is found that increasing the number of ants gives better results as it would ensure getting the optimal path instead of a sub-optimal one. Increasing the number of ants results in an increase in the probability of finding the optimal path from the 1st iteration. In addition, it results in a decrease in the probability of getting a sub-optimal path. However, increasing the number of ants results in an increase in the time to find the optimal solution.

As shown in Figure 2 and Figure 3, doubling the number of ants results in doubling the computation time needed to find the optimal solution. Moreover, the probability of finding optimal solution in the 1st iteration increased from $75 \%$ to $93 \%$ and the probability of getting a sub-optimal solution decreased from $11 \%$ to $0 \%$. 
International journal on applications of graph theory in wireless ad hoc networks and sensor networks (GRAPH-HOC) Vol.3, No.2, June 2011

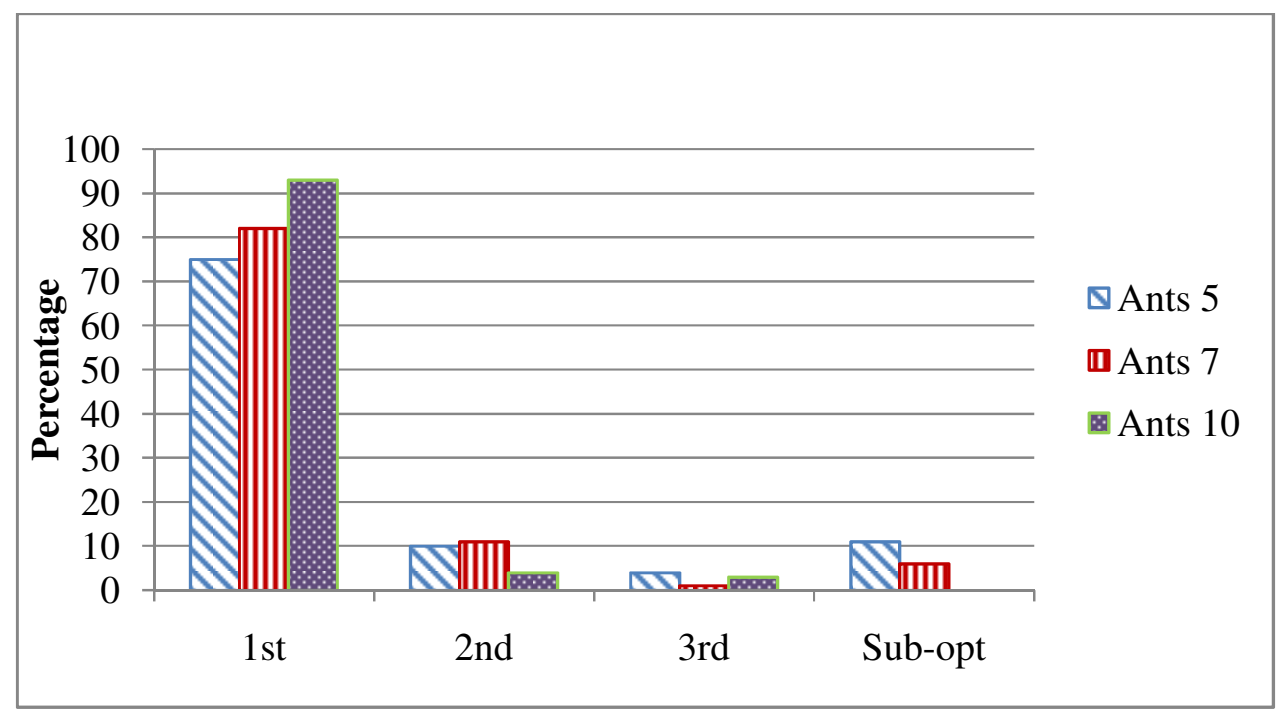

Figure 2. Effect of increasing the number of ants on rate of finding optimal path per iteration.

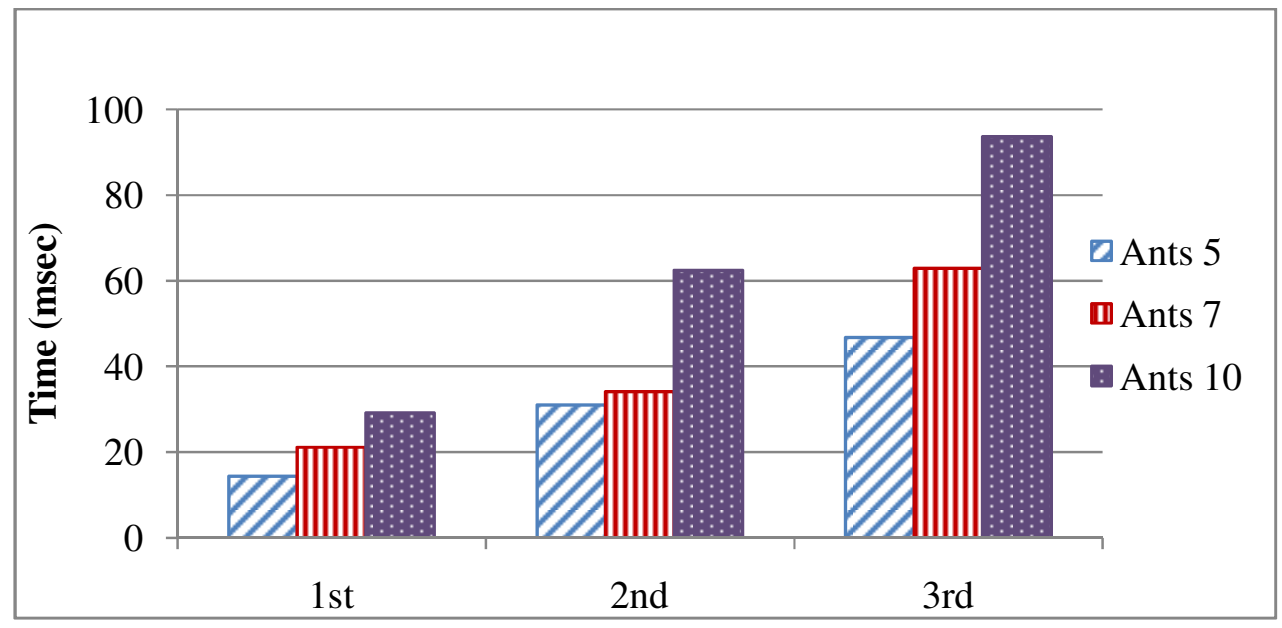

Figure 3. Effect of increasing the number of ants on computation time per iteration.

\subsection{Selection of the Pheromone Weight}

Increasing $\alpha$ yields unsatisfactory results as the solutions found may not be optimal. The reason behind this is the fact that increasing $\alpha$ means increasing the importance of following the pheromone trails very early on more than the heuristic value which is the real cost of the paths. Therefore, the probability of transition to a neighbouring node will be more influenced by the pheromone value than by the heuristic value. To find the optimal path, the heuristic value should be given a higher importance than pheromone value. 
International journal on applications of graph theory in wireless ad hoc networks and sensor networks (GRAPH-HOC) Vol.3, No.2, June 2011

\subsection{Selection of the Heuristic Weight}

In this section, the effect of increasing $\beta$ on a network with 4 nodes and on a network with 20 nodes is tested and analyzed. In addition, the effect of increasing the evaporation rate $\rho$ is tested in each case.

First, a test of increasing $\beta$ on a graph with 4 nodes where $\alpha=1$ and with different evaporation rates $\rho$ is performed as shown in Figure 4. Increasing $\beta$ from 1 to 2 when $\rho=0.2$ results in slight decrease in the probability of finding the optimal path in the first iteration but had no effect on the probability of finding a sub-optimal path. However, increasing $\beta$ to 5 results in a significant increase in the probability of finding the sub-optimal path and decreases the probability of finding the optimal one in the first iteration. Figure 4 also shows the effect of increasing $\beta$ on the same graph with 4 nodes where $\alpha=1$ and the evaporation rate is set to a higher value $\rho=0.5$. There is a slight decrease in the probability of finding the optimal path in the first iteration when $\beta$ is increased from 1 to 2 and a slight increase in the probability of finding the sub-optimal path. On the other hand, the change is significant when $\beta$ is increased to 5 .

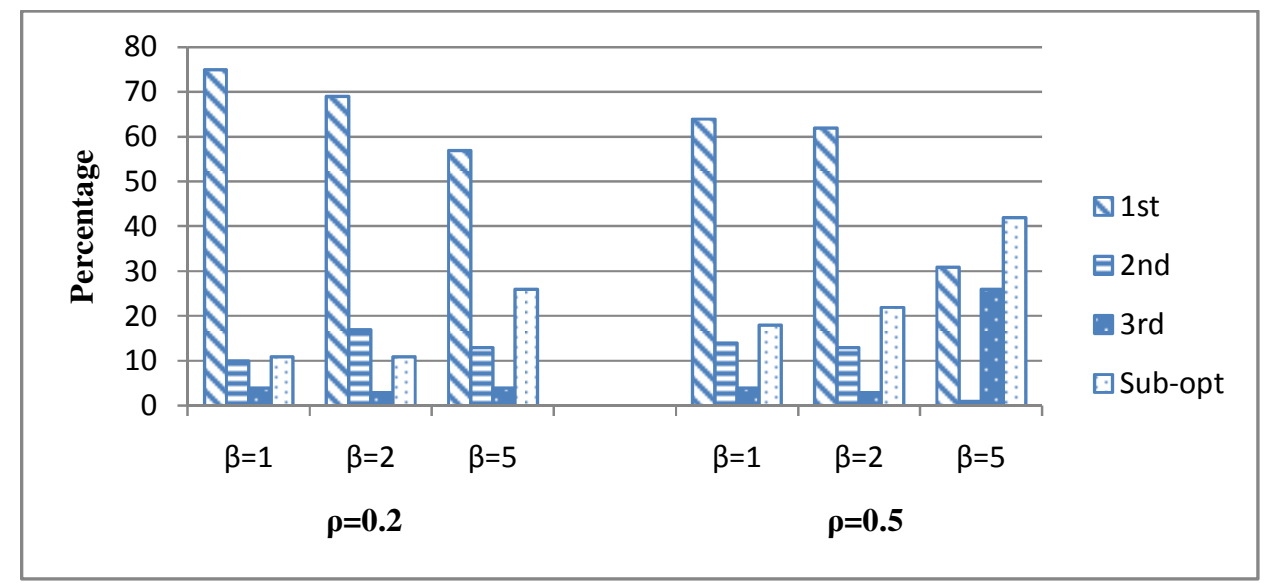

Figure 4. Effect of increasing the heuristic weight $\beta$ for $N_{S}=4$.

Figure 5 shows the effect of increasing $\beta$ on a graph with 20 nodes where $\alpha=1$ and with different evaporation rates $\rho$. In a 20-vertices graph, increasing $\beta$ from 1 to 2 results in an increase in the probability of finding the optimal path in the first iteration from $45 \%$ to $76 \%$. Moreover, it results in a decrease in the probability of finding the sub-optimal path from $18 \%$ to $13 \%$.

The best $\beta$ value found is 2 as it has the highest probability of finding optimal path in the first iteration and has the least probability of finding sub-optimal path. Increasing $\beta$ to 5 results in reaching stagnation very fast and it is evident because there are no optimal paths found in the second or third iterations. It only finds the optimal path in the first iteration with a very low probability or finds a sub-optimal path. 
International journal on applications of graph theory in wireless ad hoc networks and sensor networks (GRAPH-HOC) Vol.3, No.2, June 2011

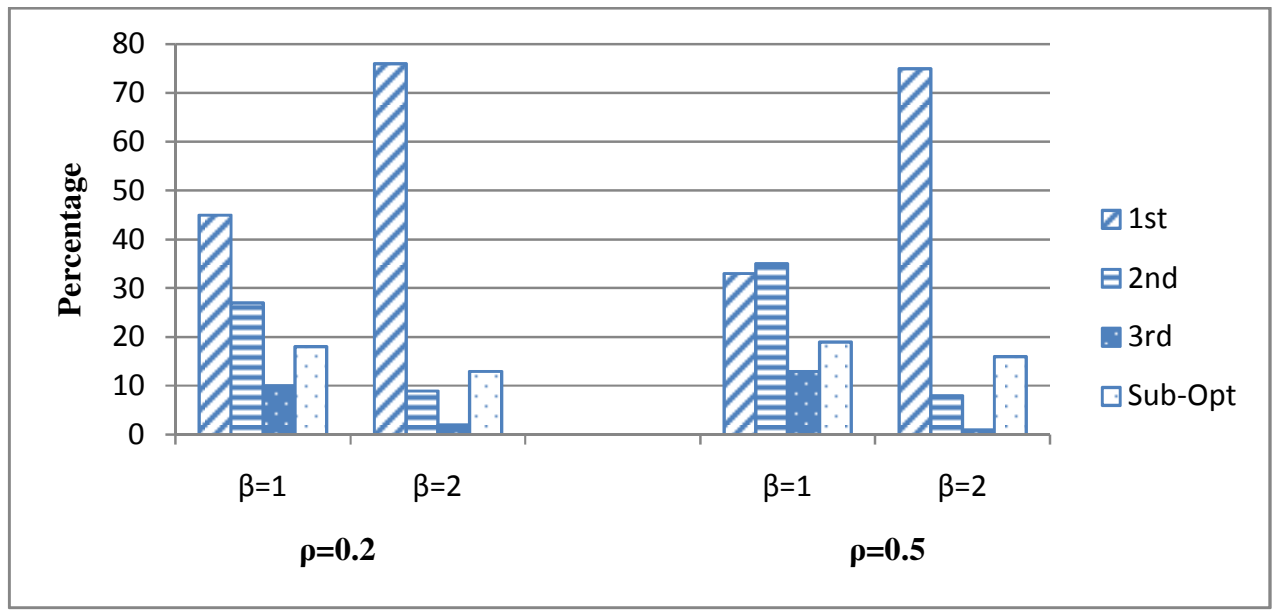

Figure 5. Effect of increasing the heuristic weight $\beta$ for $N_{S}=20$.

\subsection{Selection of the Evaporation Rate}

Figure 6 and 7 represent the effect of increasing the evaporation rate $\rho$ in 4-nodes graph where $\beta$ is 1 in Figure 6 and $\beta$ is 2 in Figure 7. Increasing $\rho$ from 0.2 to 0.5 results in decreasing the probability of finding the optimal path in the first iteration. Moreover, it results in an increase in the probability of finding the sub-optimal paths. However, the effect is minimal when $\beta$ is 2 .

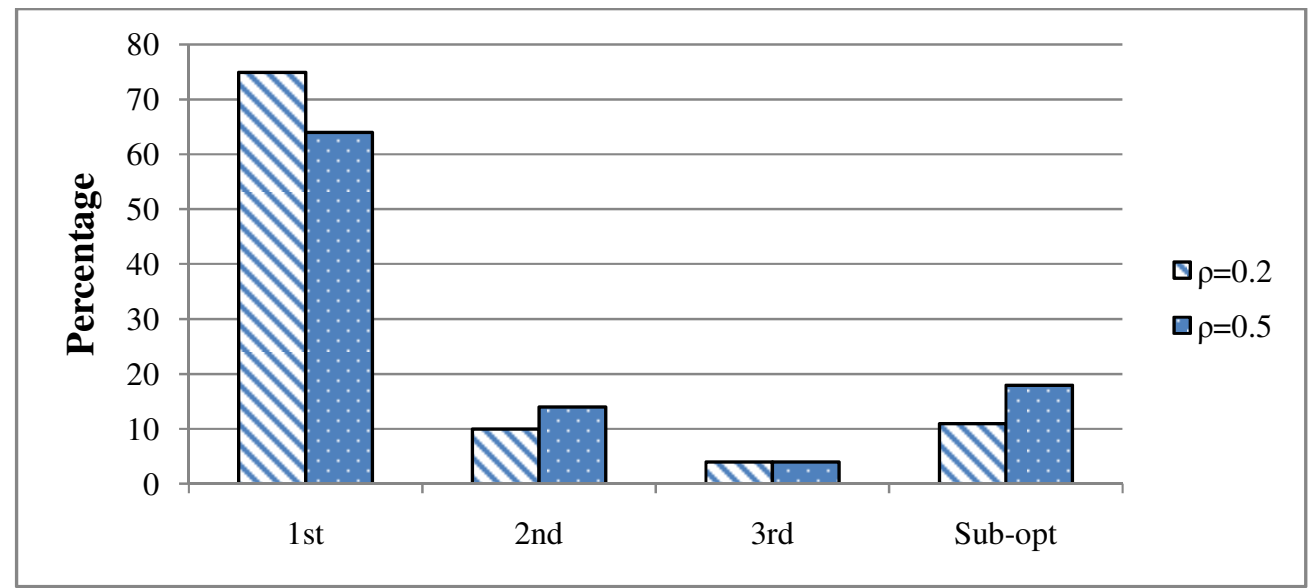

Figure 6. Effect of increasing the evaporation rate $\rho$ for $N_{S}=4, \alpha=1, \beta=1$. 
International journal on applications of graph theory in wireless ad hoc networks and sensor networks

(GRAPH-HOC) Vol.3, No.2, June 2011

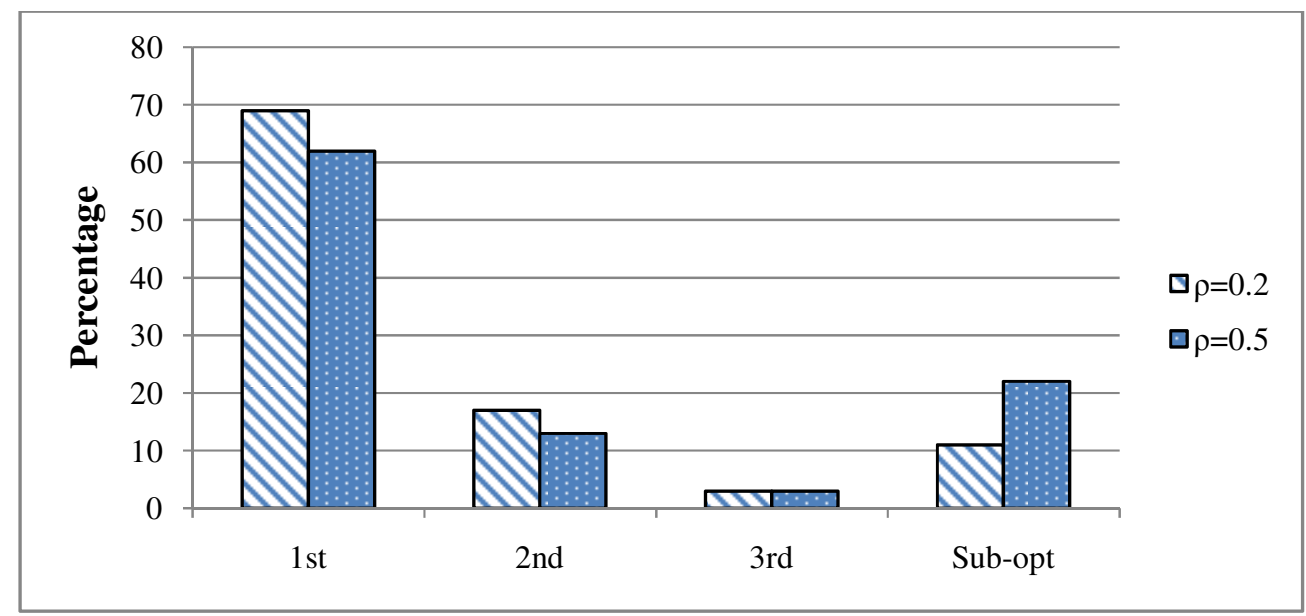

Figure 7. Effect of increasing the evaporation rate $\rho$ for $N_{S}=4, \alpha=1, \beta=2$.

Figure 8 and 9 show the effect of increasing the evaporation rate $\rho$ in 20-nodes graph where $\beta$ is 1 in Figure 8 and $\beta$ is 2 in Figure 9. Increasing evaporation rate $\rho$ would follow the same pattern mentioned in 4-nodes graph which is a decrease in the probability of finding the optimal path in the first iteration and an increase in sub-optimal probability. However, increasing suboptimal probability has less effect in 20-nodes graph compared to 4-nodes graph. Moreover, as shown in Figure 9, the effect of increasing the evaporation rate $\rho$ when $\beta$ is 2 is much less when compared to 4-vertices graph.

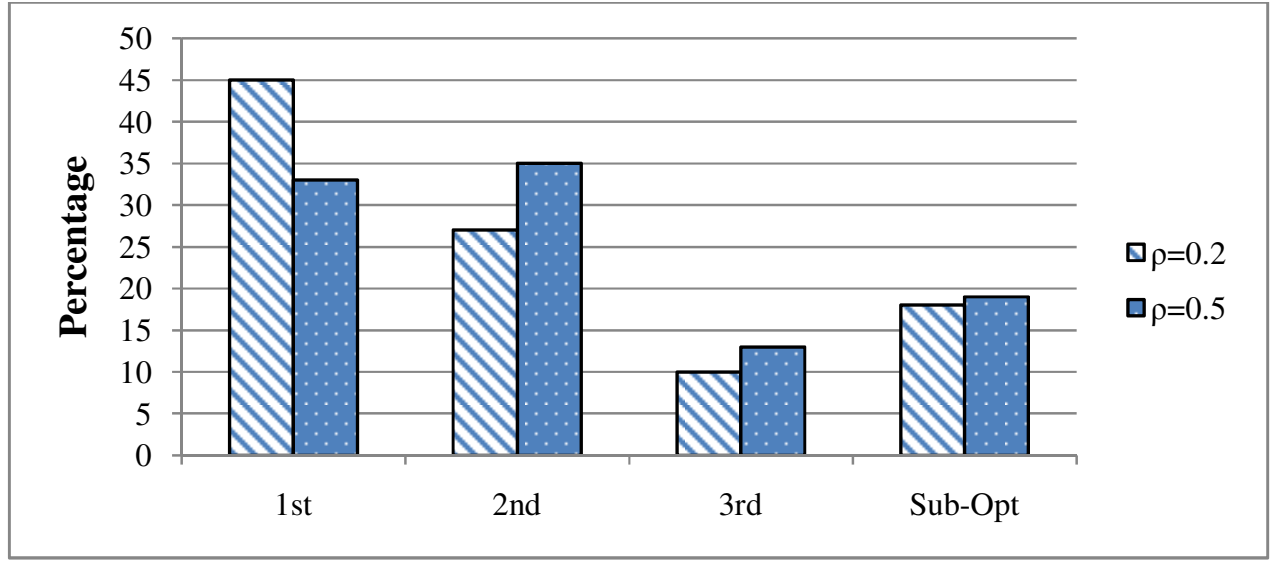

Figure 8. Effect of increasing the evaporation rate $\rho$ for $N_{S}=20, \alpha=1, \beta=1$. 
International journal on applications of graph theory in wireless ad hoc networks and sensor networks (GRAPH-HOC) Vol.3, No.2, June 2011

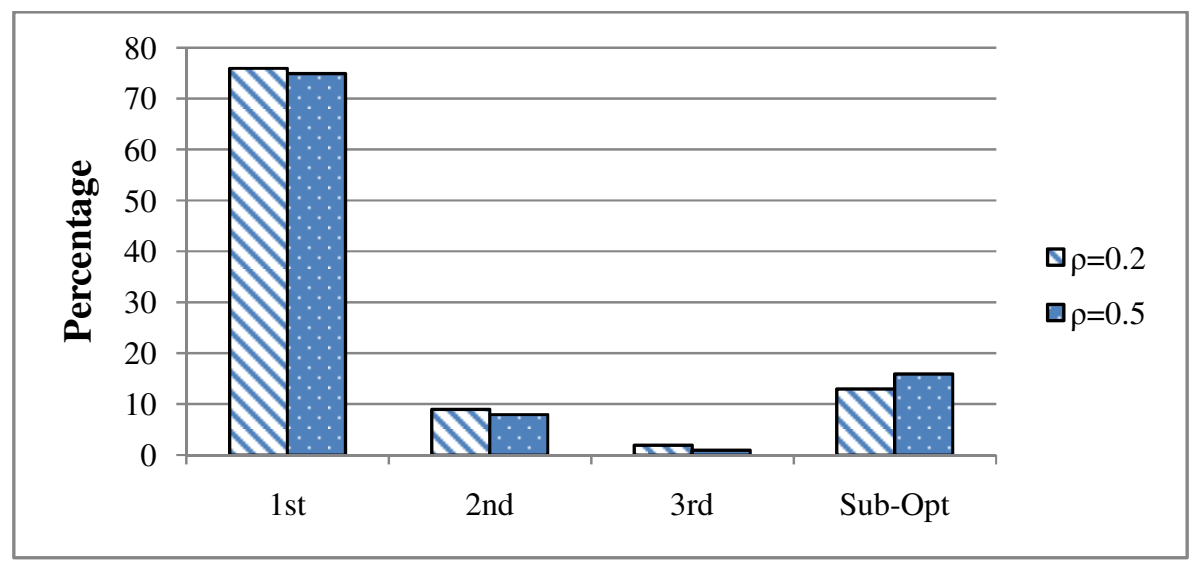

Figure 9. Effect of increasing the evaporation rate $\rho$ for $N_{S}=20, \alpha=1, \beta=2$.

\section{Analysis of Ant Colony-BaSed Routing in Multimedia WSN}

\subsection{Analysis of the Transmission Rate}

In this study, all simulation parameters are fixed and the effect of increasing transmission bit rate $R_{b}$ is evaluated. Event generation rate $\lambda$ is set to be equal to one event per second and the video frame encoding rate $F_{p}$ is set to be equal to 10 frames per second.

Figure 10 represents the effect of increasing transmission bit rate on average queuing delay per node and it is clear that by increasing $R_{b}$, the average queuing delay is reduced significantly. Subsequently, the loss percentage per event will decrease as shown in Figure 11.

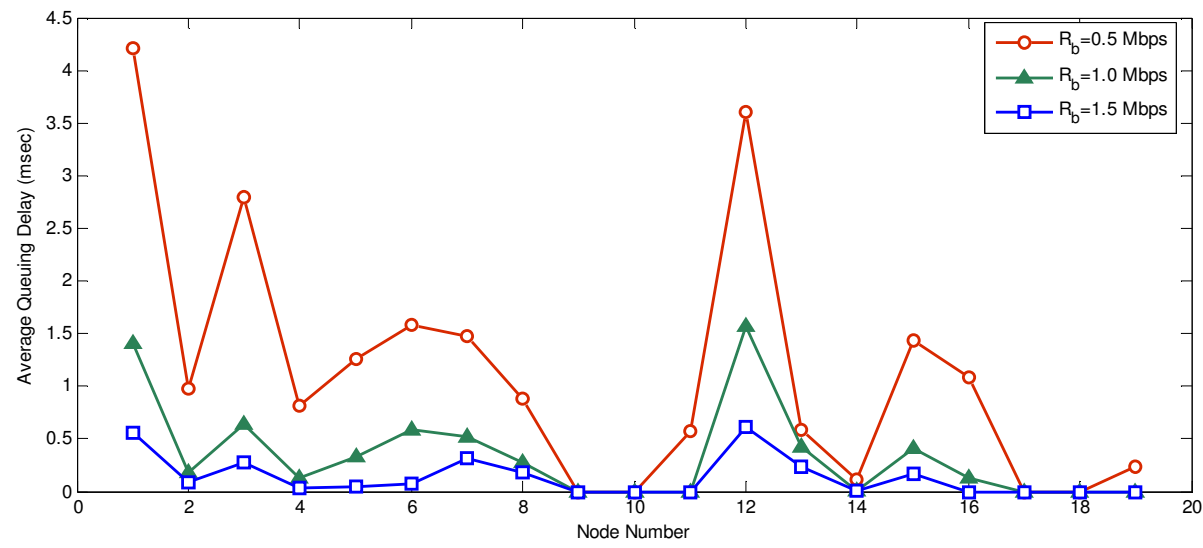

Figure 10. Effect of varying the transmission bit rate on average queuing delay per sensor node. 
International journal on applications of graph theory in wireless ad hoc networks and sensor networks (GRAPH-HOC) Vol.3, No.2, June 2011

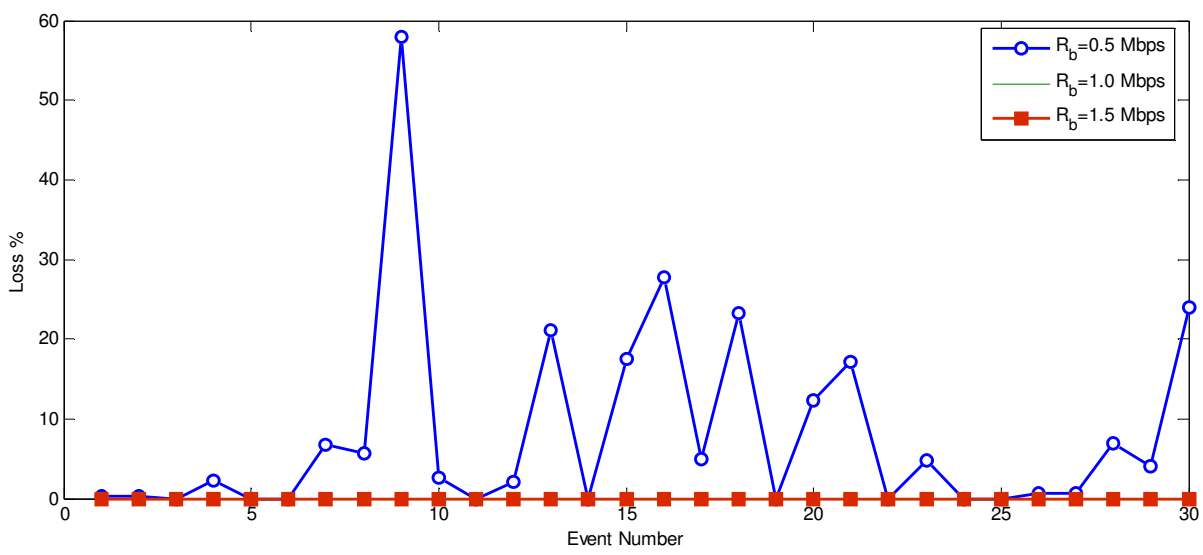

Figure 11. Effect of varying the transmission bit rate on frame loss percentage per event.

Figure 12 shows the end-to-end delay per video frame and as $R_{b}$ increases, the end-to-end delay decreases. This is due to the fact that the transmission delay is inversely proportional to $R_{b}$ and thus when $R_{b}$ increases, transmission delay decreases. In addition, the average queuing delay per sensor node also decreases due to faster transmission of packets. Figure 13 shows the delay jitter decreases as $R_{b}$ increases. In addition, the variation in delay jitter between frames is reduced when $R_{b}$ increases.

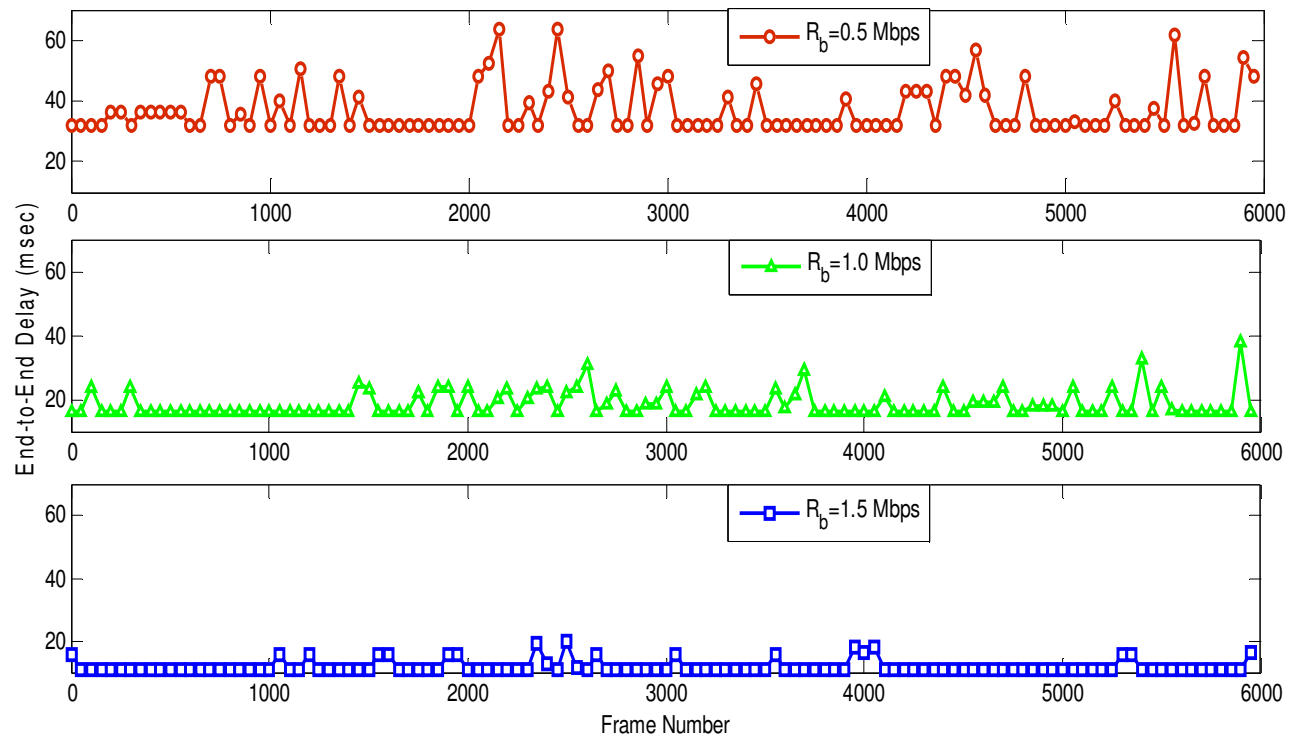

Figure 12. Effect of varying the transmission bit rate on end-to-end delay per packet. 

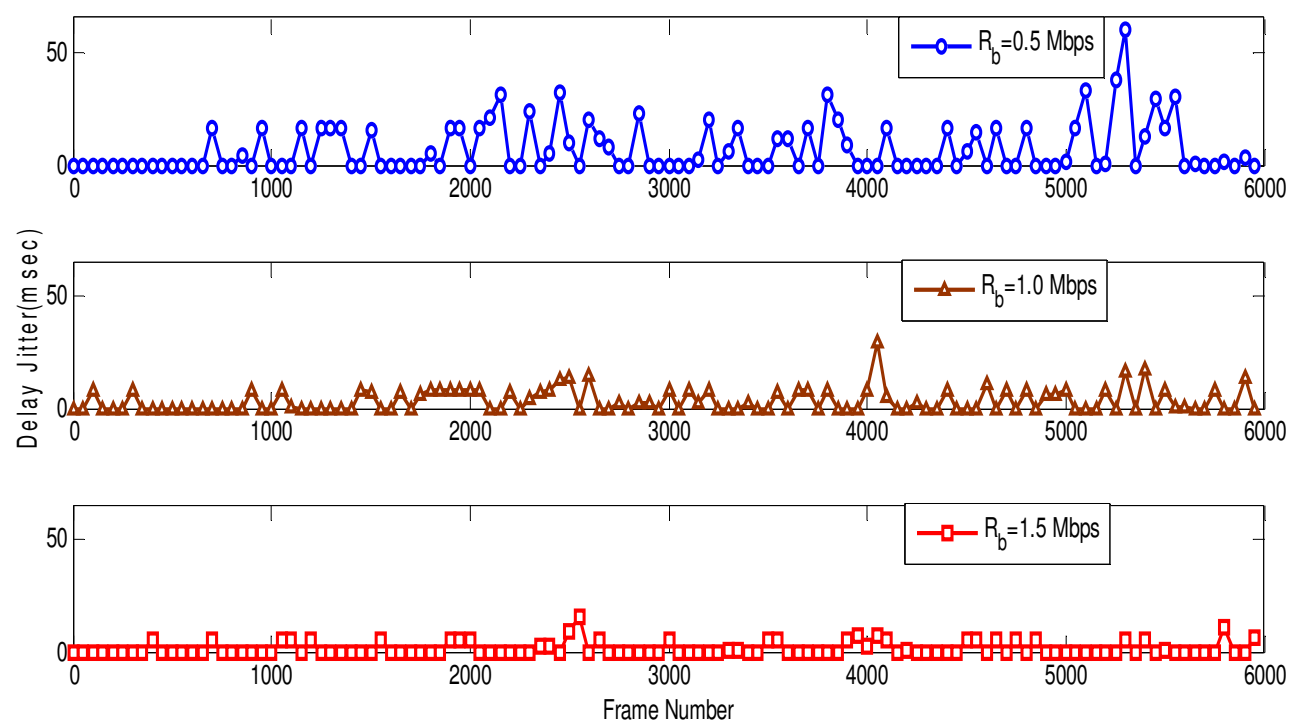

Figure 13. Effect of varying the transmission bit rate on delay jitter per packet.

\subsection{Analysis of the Event Generate Rate}

In this study, all simulation parameters are fixed and the effect of increasing event generation rate is evaluated. Transmission bit rate is set to be equal to $0.5 \mathrm{Mbps}$ and video frame encoding rate is set to be equal to 10 frames per second.

As shown in Figure 14, increasing event generation rate results in increasing average queuing delay. This leads to delaying many packets beyond the deadline set and results in increasing loss percentage per event as shown in Figure 15.

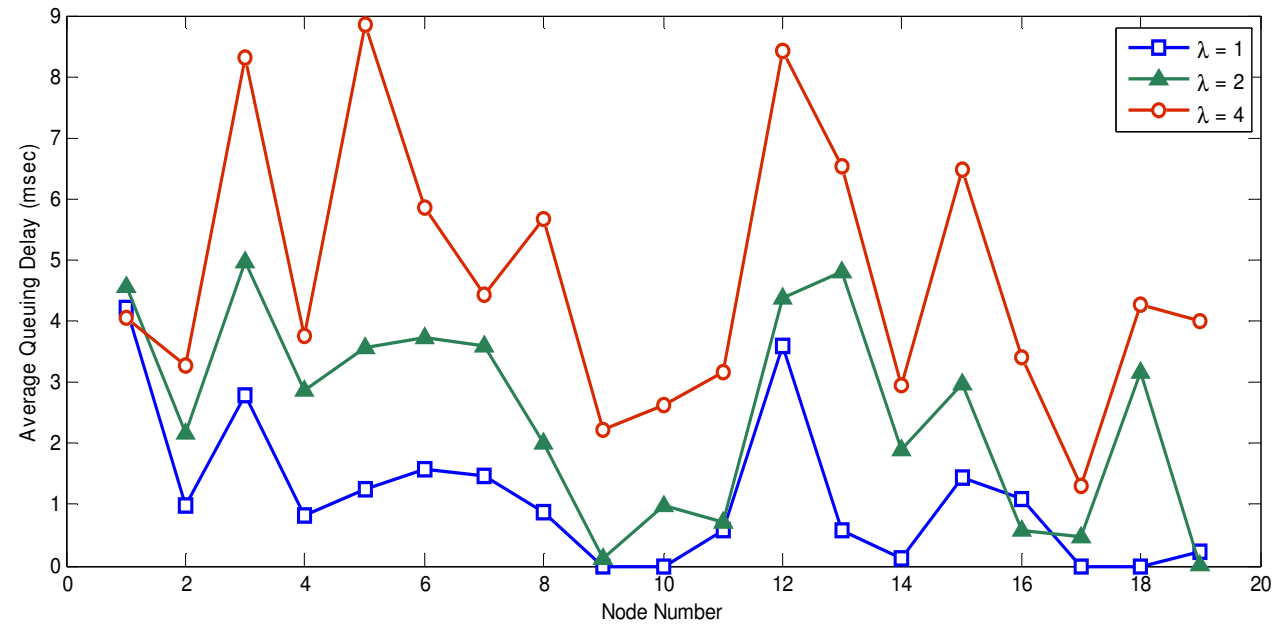

Figure 14. Effect of $\lambda$ on average queuing delay per sensor node 
International journal on applications of graph theory in wireless ad hoc networks and sensor networks (GRAPH-HOC) Vol.3, No.2, June 2011

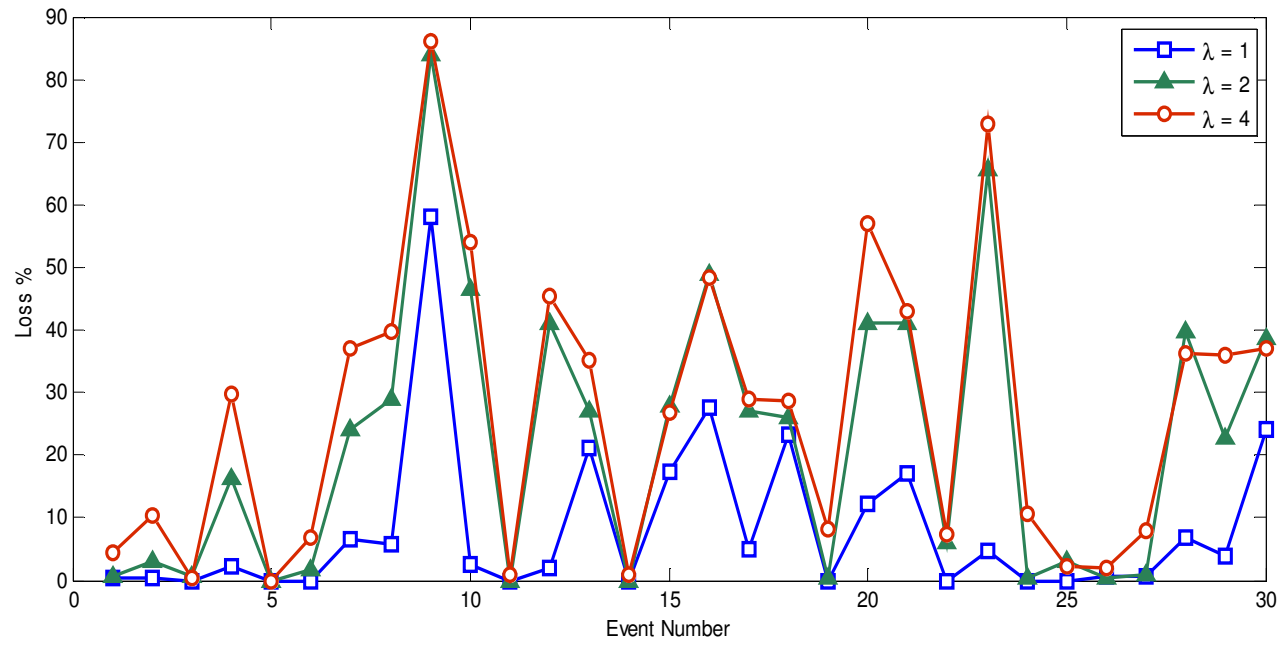

Figure 15. Effect of $\lambda$ on loss percentage per event

Figure 16 shows the end-to-end delay per packet where the end-to-end delay per packet increases as increases due to the increase in queuing delay and the variation in delay jitter increases as shown in Figure 17.

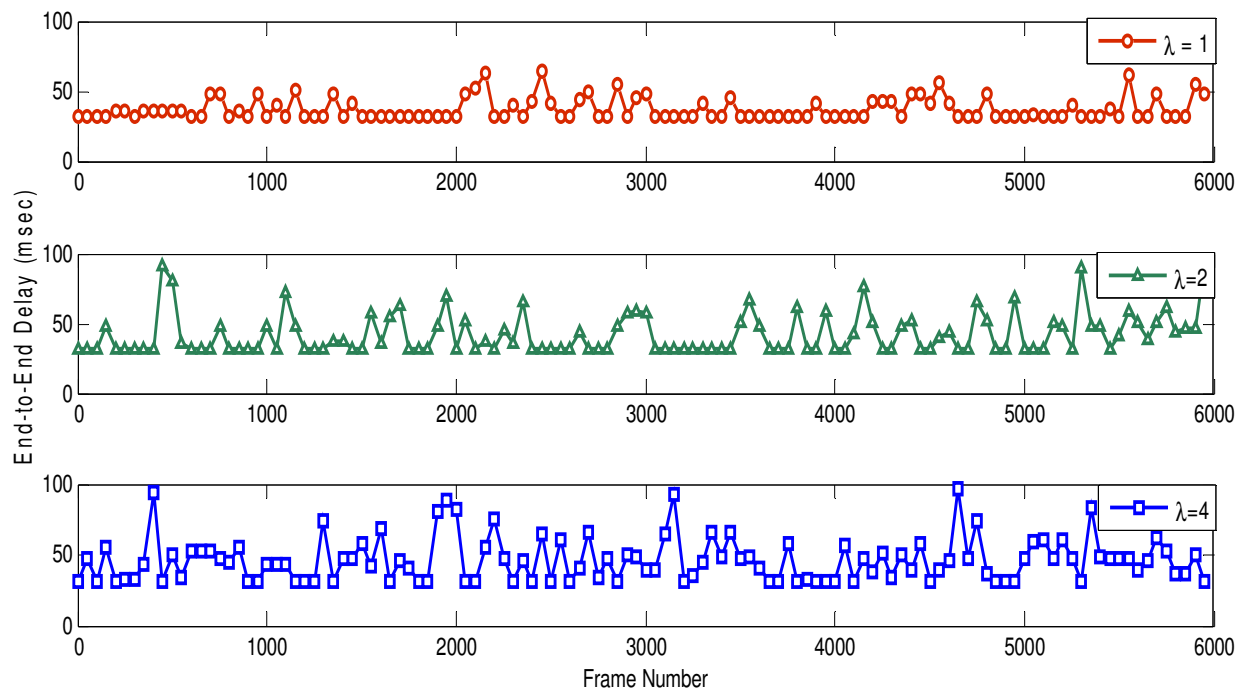

Figure 16. Effect of $\lambda$ on end-to-end delay per packet 
International journal on applications of graph theory in wireless ad hoc networks and sensor networks

(GRAPH-HOC) Vol.3, No.2, June 2011
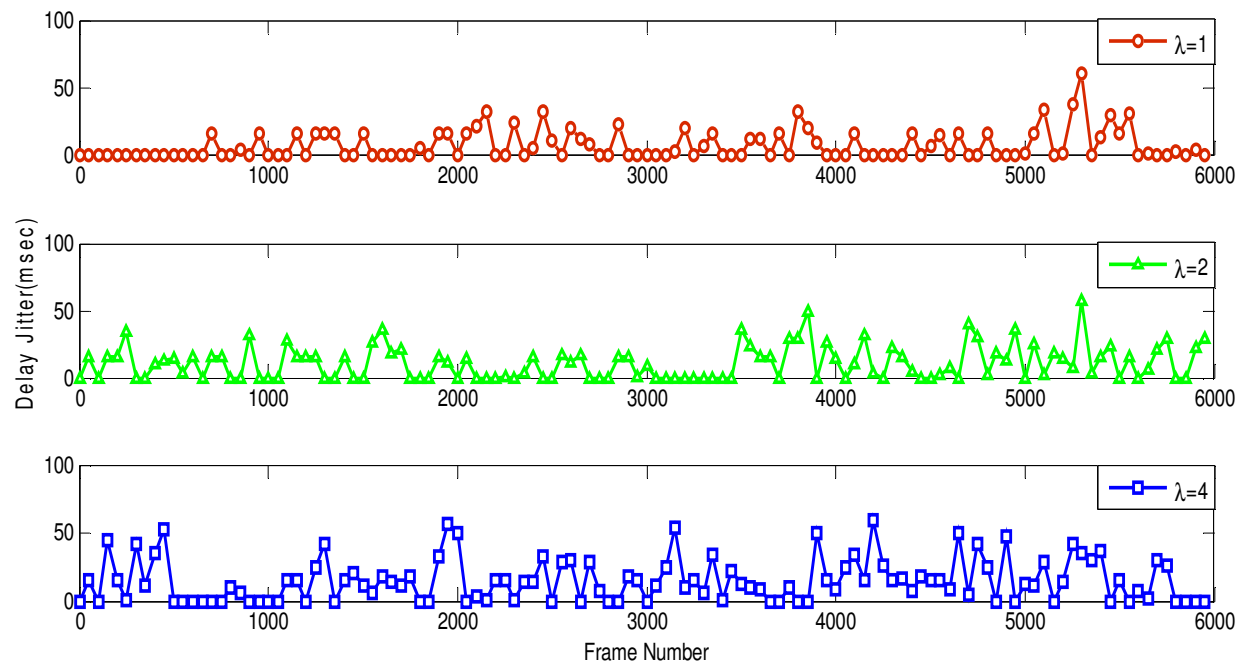

Figure 17. Effect of $\lambda$ on delay jitter per packet

\subsection{Analysis of the Video Encoding Rate}

In this study, all simulation parameters are fixed and the effect of increasing video frame encoding rate is evaluated. Event generation rate is set to be equal to 1 event per second and transmission bit rate is set to be equal to $0.5 \mathrm{Mbps}$.

In Figure 18, the average queuing delay increases with the increase of video frame encoding rate in most of the nodes. While in other nodes, the average queuing delay decreases or stays the same and this is because after reaching certain average queuing delay during simulation, the sensor node would drop packets which are expected to miss the deadline based on the estimation of remaining queuing and transmission delay in the path. When the loss percentage per event increases, the nodes' average queuing delay decreases or stays the same. Figure 19 shows the large loss percentage increase while increasing frame encoding rate.

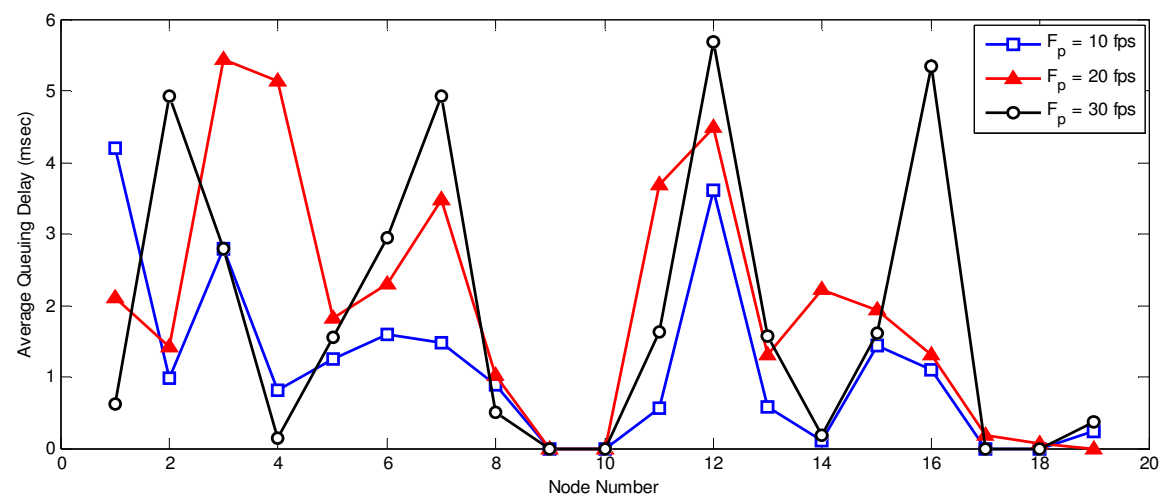

Figure 18. Effect of frame generation rate $F_{p}$ on average queuing delay per sensor node. 
International journal on applications of graph theory in wireless ad hoc networks and sensor networks

(GRAPH-HOC) Vol.3, No.2, June 2011

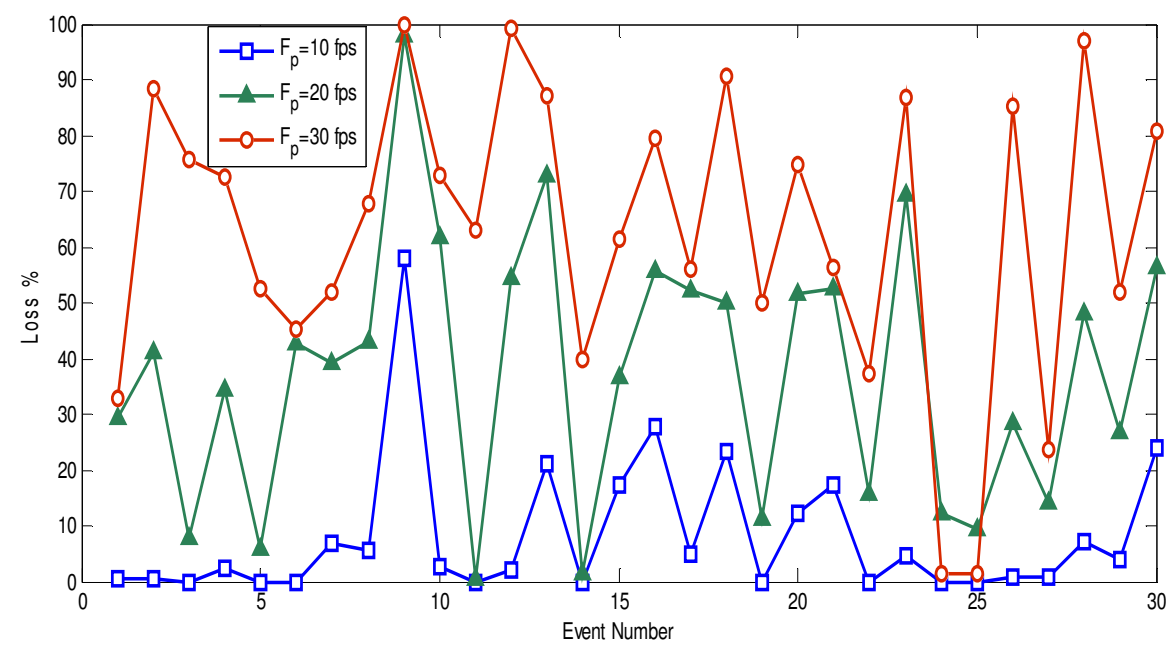

Figure 19. Effect of frame generation rate $F_{p}$ on loss percentage per event.

Figure 20 shows the end-to-end delay per packet and Figure 21 shows the delay jitter. It is noticeable that the end-to-end delay decreased when frame encoding rate is 30 frames/s. However, this decrease is the result of the large loss percentage which results in either transmitting packets with very low queuing delay or dropping packets. The end-to-end delay when for frame generation rate of 30 frames/s is equal to transmission delay for most of the packets and there are only few packets experienced a queuing delay as shown in Figure 20. At a transmission bit rate equal to $0.5 \mathrm{Mbps}$, generating 30 frames per second results in high loss and therefore is not acceptable. In order to generate at 30 frames per second, the transmission bit rate should be higher than 0.5 Mbps.

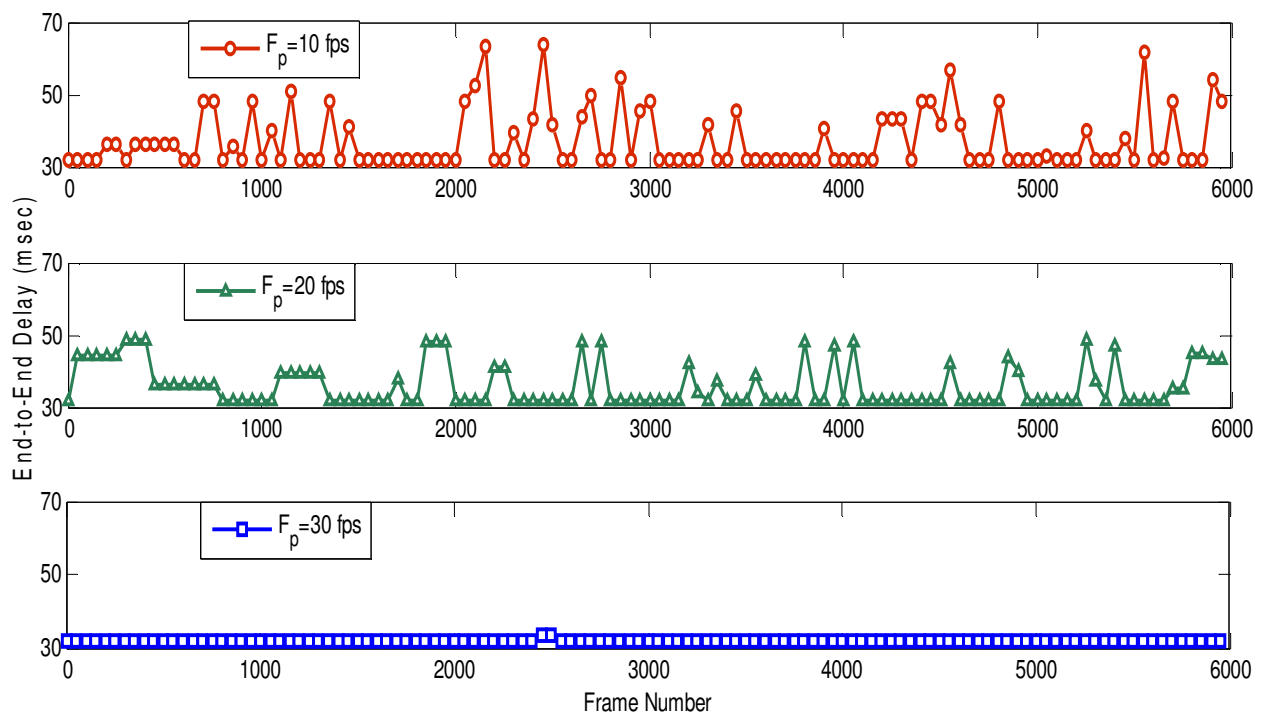

Figure 20. Effect of frame generation rate $F_{p}$ on end-to-end delay per packet. 
International journal on applications of graph theory in wireless ad hoc networks and sensor networks

(GRAPH-HOC) Vol.3, No.2, June 2011
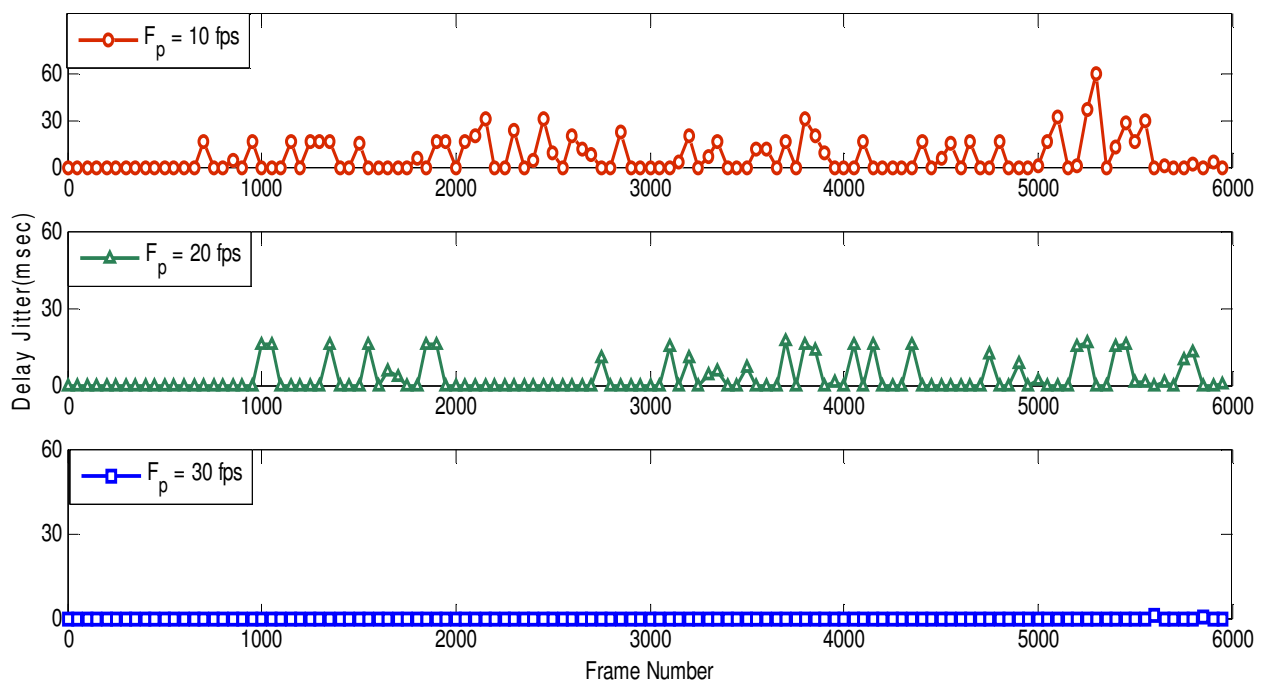

Figure 21. Effect of frame generation rate $F_{p}$ on delay jitter per packet.

\section{CONCLUSIONS}

In this paper, we propose an ant colony-based routing algorithm for multimedia WSNs. This algorithm optimizes energy consumption, link quality and link reliability. The energy consumption used considers communication energy cost, transmission energy cost, and the sensor node's energy level. This results in choosing the optimal path that minimizes energy consumption and prolongs the lifetime of the network. Moreover, the optimal path has a high link quality and reliability. Depending on the application, different weights are given to energy consumption, link quality and link reliability.

A study of this algorithm is performed on different network sizes and this algorithm is further analyzed for the effect of changing the number of ants, pheromone value importance, the heuristic value importance, and the evaporation rate on the probability of finding the optimal path and the time taken to find it. Moreover, a simulation of multimedia WSN that uses the proposed ant colony optimization is performed. The effects of transmission bit rate, event generation rate, and frame encoding rate are examined.

\section{ACKNOWLEDGEMENTS}

The authors would like to thank the College of Engineering at the American University of Sharjah for its support of this research. 
International journal on applications of graph theory in wireless ad hoc networks and sensor networks

(GRAPH-HOC) Vol.3, No.2, June 2011

\section{REFERENCES}

[1] J. Yick, B. Mukherjee, D. Ghosal, "Wireless sensor network: survey," Comput. Networks (Elsevier), vol. 52, no. 12, pp. 2292-2330, Aug. 2008.

[2] S.Tarannum, "Energy conversation challenges in wireless senor networks: a comprehensive study," Wireless Sensor Networks, vol. 2, no. 6, pp. 483.

[3] I.F. Akyildiz, T. Melodia, KR. Chowdhury, "A survey on wireless multimedia sensor networks," Comput. Networks, vol. 51, no. 4, pp. 921-960, Nov. 2007.

[4] E. Gurses, O.B. Akan, "Multimedia communication in wireless sensor networks," Ann. Telecommun., vol. 60, no. 7-8, pp. 799-827, 2005.

[5] S. Misra, M. Reisslein, G. Xue, "A survey of multimedia streaming in wireless sensor networks," IEEE Commun. Surveys \& Tutorials, vol. 10, no. 4, pp.18-39, 2008.

[6] M. Abolhasan, T. Wysocki, and E. Dutkiewicz, "A review of routing protocols for mobile ad hoc networks," Ad Hoc Networks (Elsevier), vol. 2. pp. 1-22, 2004.

[7] K. Akkaya, M. Younis, "A survey of routing protocols in wireless sensor networks," Ad Hoc Network (Elsevier), vol. 3, no. 3, pp. 325-349, 2005

[8] J.N. Al-Karaki, A.E. Kamal, "Routing techniques in wireless sensor networks: a survey," IEEE Wireless Commun., vol. 11, no. 6, pp. 6-28, 2004.

[9] M. Abdul Hamid, M. Alam, C. Hong, "Design of a QoS-aware routing mechanism for wireless multimedia sensor networks," IEEE Proc. Global Telecomm. Conf. (GLOBECOM), New Orleans, LO, pp.1-6, Dec. 2008.

[10] J. Martínez, A. García, I. Corredor, L. López, Vicente Hernández, A. Dasilva, "QoS in wireless sensor network: survey and approach," Proc. Of the 2007 Euro American Conf. on Telematics and inform. Syst. (EATIS 2007).

[11] C. Shasha, K. Zongwu, C. Niansheng, "Research of QoS routing technology for wireless multimedia sensor network," IEEE Int. Colloq. on Computing, Commun., Control, and Management (ISECS 2009), Sanya, China, pp. 334-337, Aug. 2009.

[12] B. Bhuyan, H. Sarma, N. Sarma, A. Kar, R. Mall, "Quality of service provision in wireless sensor networks and related challenges," Wireless Sensor Network, vol.2, pp.861-868, Nov. 2010.

[13] N. Zhang, A. Anpalagan, "Comparative review of QoS-aware on-demand routing in ad hoc wireless networks,”Wireless Sensor Network, pp.274-284, 2010.

[14] K. Sohrabi, J. Pottie, "Protocols for self-organization of a wireless sensor network", IEEE Pers. Commun., vol. 7, no. 5, pp. 16-27, 2000

[15] T. He, J. Stankovic, C. Lu, T. Abdelzaher, "SPEED: a stateless protocol for real-time communication in sensor networks,” IEEE Proc. Int. Conf. Distributed Computing Syst., pp. 46-55, 2003.

[16] E. Felemban, C.-G. Lee, E. Ekici, "MMSPEED: Multipath multi-SPEED protocol for QoS guarantee of reliability and timeliness in wireless sensor networks, IEEE Trans. Mobile Comput. , vol. 5, no. 6, pp. 738754,2006

[17] K. Akkaya, M. Younis, "An energy-aware QoS routing protocol for wireless sensor networks," in: Proc. of Int. Conf. on Distributed Computing Syst. Workshops (ICSDSW), Washington, DC, 2003.

[18] J. Zhu, H. Zhao, J. Xu, "An energy balanced reliable routing metric in WSNs", Wireless Sensor Networks, vol. 1, no. 1, pp. 22, 2009.

[19] M. Dorigo, T. Stutzle, “Ant colony optimization,” Bradford Book, 2004.

[20] T. Camilo, C. Carreto, J. Silva, and F. Boavida, "An energy-efficient ant-based routing algorithm for wireless sensor networks," Ant Colony Optimization and Swarm Intell., Lecture Notes in Comput. Sci., vol. 4150, pp. $49-59,2006$

[21] S. Okdem, and D. Karaboga, "Routing in wireless sensor networks using ant colony optimization," 1st NASA/ESA Conf. on Adaptive Hardware and Systems (AHS), Istanbul, Turkey, pp. 401-404, 2006. 
International journal on applications of graph theory in wireless ad hoc networks and sensor networks

(GRAPH-HOC) Vol.3, No.2, June 2011

[22] S. Okdem, D. Karaboga, "Routing in wireless sensor networks using an ant colony optimization (ACO) router chip," Sensors, vol. 9, pp. 909-921, 2009.

[23] K. Saleem, N. Fisal, S. Hafizah, S. Kamilah, R.A. Rashid, “A self-optimized multipath routing protocol for wireless sensor networks,” Intl. J. of Recent Trends in Eng., vol. 2, no. 1, pp. 93-97, Nov. 2009.

[24] R. G. GhasemAghaeil, Md. Abdur Rahman, W. Gueaieb, and A. El Saddik, "Ant Colony-Based Reinforcement Learning Algorithm for Routing in Wireless Sensor Networks," IEEE Int. Instrumentation and Measurement Technology Conf. (IMTC 2007), Warsaw, Poland, May 2007.

[25] Md. Abdur Rahman, R. GhasemAghaeil, A. El Saddik, and W. Gueaieb, "M-IAR: biologically inspired routing protocol for wireless multimedia sensor networks," IEEE Int. Instrumentation and Measurement Technology Conf., Vancouver Island, Canada, May 2008.

[26] L. Cobo, A. Qintero, S. Pierre, “ Ant-based routing for wireless multimedia sensor networks using multiple QoS metrics," Computer Networks, vol. 54.

[27] W. Heinzelman, A. Chandrakasan and H. Balakrishnan, "Energy-efficient communication protocol for wireless microsensor networks," Proc. of the 33rd Int. Conf. on Syst. Sci. (HICSS '00), 2000.

[28] W. Heinzelman, A. Chandrakasan, and H. Balakrishnan, "An application-specific protocol architecture for wireless microsensor networks," IEEE Trans. Wireless Commun., vol. 1, no. 4, pp. 660-670, Oct. 2002.

[29] X. Haung, Y. Fang, "Multiconstrained QoS multipath routing in wireless sensor networks," Wireless Network, vol. 14, pp.465-478, 2007.

[30] D. Cheng, Y. Xun, T. Zhou and W. Li, "An Energy Aware Ant Colony Algorithm for the Routing of Wireless Sensor Networks," Springer's Journal on Communications in Computer and Information Science, Volume 134, 395-401, 2011.

[31] Pavai, K.; Sivagami, A.; Sridharan, D. , "Study of Routing Protocols in Wireless Sensor Networks," International Conference on Advances in Computing, Control, \& Telecommunication Technologies, 2009. ACT '09., pp.522-525, 28-29, Dec. 2009

[32] K. S. Low, W. N. Win, and M. J. Er, " Wireless Sensor Networks for Industrial Environments", In proceedings of the IEEE International Conference on CIMCA-IAWTIC, 2005.

[33] Alsaify, B.A.; Thompson, D.R.; , "Pendulum: An energy efficient protocol for Wireless Sensor Networks," Sensors Applications Symposium (SAS), 2010 IEEE , pp.273-277, 23-25 Feb. 2010

[34] Gui, N.; Ruichuan Chen; Zhuhua Cai; Jianbin Hu; Zhong Chen; , "A Secure Routing and Aggregation Protocol with Low Energy Cost for Sensor Networks," Information Engineering and Electronic Commerce (IEEC '09), pp.79-84, 16-17 May 2009.

[35] Huan Gong; Jianxin Wang; , "Study of energy consumption problem in wireless sensor networks," 2010 International Conference on Mechanic Automation and Control Engineering (MACE), pp.4559-4561, 26-28 June 2010 .

[36] N. Meghanathan, "Survey and Taxonomy of Unicast Routing Protocols for Mobile Ad-Hoc Networks", Int. J. on App. of Graph Theory in Wireless Ad hoc Networks and Sensor Networks, Vol. 1, No. 1, pp. 1-21, 2009.

[37] A. Gowri, et al., "A Review: Optimal Path Selection in Ad hoc Networks using Fuzzy Logic ”, Int. J. on App. of Graph Theory in Wireless Ad hoc Networks and Sensor Networks, Vol. 2, No. 4, pp. 1-6, 2010.

[38] B. Olagbegi and N. Meghanathan, "A Review of the Energy Efficient and Secure Multicast Routing Protocols for Mobile Ad Hoc Networks", Int. J. on App. of Graph Theory in Wireless Ad hoc Networks and Sensor Networks, Vol. 2, No. 2, pp. 1-15, 2010.

[39] S. Ali, K. Al-Omari and P. Sumari, "An Overview of Mobile Ad Hoc Networks for the Existing Protocols and Applications", Int. J. on App. of Graph Theory in Wireless Ad hoc Networks and Sensor Networks, Vol. 2, No. 1, pp. 87-110, 2010.

[40] D. S. Devi and G. Padmavathi, "Impact of Mobility for Qos Based Secure Manet", Int. J. on App. of Graph Theory in Wireless Ad hoc Networks and Sensor Networks, Vol. 2, No. 3, pp. 46-57, 2010. 